\title{
Spatial prediction of seabed sediment texture classes by cokrig- ing from a legacy database of point observations.
}

\author{
R.M. Lark ${ }^{1}, *$, D. Dove ${ }^{2}$, S.L. Green ${ }^{2}$, A.E. Richardson ${ }^{2}$, H. Stewart ${ }^{2}$, A. Stevenson ${ }^{2}$ \\ ${ }^{1}$ British Geological Survey, Keyworth, Nottinghamshire NG12 5GG, U.K. and ${ }^{2}$ British Geo- \\ logical Survey, Murchison House, Edinburgh EH9 3LA U.K. \\ * Corresponding author. Tel: +44(0)1159363026 Email address: mlark@bgs.ac.uk
}

\begin{abstract}
This paper illustrates the potential for statistical mapping of seabed sediment texture classes. It reports the analysis of legacy data on the composition of seabed sediment samples from the UK Continental Shelf with respect to three particle size classes (sand, mud, gravel). After appropriate transformation for compositional variables the spatial variation of the sediment particle size classes was modelled geostatistically using robust variogram estimators to produce a validated linear model of coregionalization. This was then used to predict the composition of seabed sediments at the nodes of a fine grid. The predictions were backtransformed to the original scales of measurement by a Monte Carlo integration over the prediction distribution on the transformed scale. This approach allowed the probability to be computed for each class in a classification of seabed sediment texture, at each node on the grid. The probability of each class, and derived information such as the class of maximum probability could therefore be mapped. Predictions were validated at a set of 2000 randomly sampled locations. The class of maximum probability corresponded to the observed class with a frequency of 0.7 , and the uncertainty of this prediction was shown to depend on the absolute probability of the class of maximum probability. Other tests showed that this geostatistical approach gives reliable predictions with meaningful uncertainty measures. This provides a basis for rapid mapping of seabed sediment texture to classes with sound quantification of the uncertainty. Remapping to revised class definitions can also be done rapidly, which will be of particular value in habitat mapping where the seabed geology is an important factor in biotope modelling.
\end{abstract}

Keywords: Seabed sediment; texture classes; habitat mapping; cokriging; compositional data 


\section{Introduction}

Seabed and habitat mapping is important for a range of activities in the marine environment, including fisheries, aquaculture, conservation, aggregate extraction, renewable energy, seabed infrastructure and the extraction of oil and gas. Conservation organizations, resource managers, marine spatial planners and policy-makers need to understand seabed habitats. As a result, benthic habitat mapping is a growing focus of activity for scientists, driven by scientific, economic and political factors (Harris and Baker, in press). The composition of the substrate is recognized as an important property to map, not least because of its importance in determining the distribution of benthic marine organisms, and its value as a proxy variable in habitat mapping and the assessment of biotopes (Connor et al., 2006; Howell, 2010; Cameron and Askew, 2011). Geoscientists are therefore producing substrate maps to assist habitat mapping, and are using a variety of methods to do so. These include classical hand interpretation in which several geophysical and geotechnical datasets are integrated; and the semi-automated interpretation of geophysical datasets.

The Marine Environmental Mapping Programme (MAREMAP) of the United Kingdom Natural Environment Research Council (NERC) is concerned with combined seabed and habitat mapping at national scale, integrating existing data sets and exploiting new technologies. Among the data sets available is the British Geological Survey's (BGS) database on seabed sediments and their particle size distribution, collected in a series of surveys from 1967 to 2009. Typically these sediment samples have been used in conjunction with geophysical data to produce regional seabed sediment and shallow geological maps and interpretations (Cameron et al., 1992; Gatliff et al., 1994). Such traditional geological mapping is valuable. However, since the legacy data are extensive, there is the potential to use statistical methods for spatial prediction to map seabed texture continuously or according to established classifications. This is potentially useful for three reasons. First, statistical mapping provides a quantitative account of the uncertainty in the predictions, which is inevitable given the spatial variability of the phenomena we are considering. Second, a statistical approach to mapping can be semi-automated, at least with respect to the generation of spatial predictions after the initial statistical modelling. This is useful because it means that maps can be revised relatively easily. The classification schemes that best predict benthic habitats are regularly being refined and improved, and statistical mapping, as described in this 
paper, can be used to generate maps according to modified classifications in a relatively short time frame. Third, statistical mapping from the extensive data available allows us to generate maps rapidly. EU legislation, such as the Marine Strategy Framework Directive and the Habitats and Species Directive increase the requirement for broad-scale mapping, covering the UK Continental Shelf. Recent reviews suggest just $10 \%$ of the UKCS habitat map coverage is derived from survey data (Department for Environment, Food and Rural Affairs, 2010). Because of this, it is extremely valuable to have semi-automated statistical mapping methods to underpin habitat prediction.

This paper is concerned with how a set of point observations can be used to map the spatial variations of seabed texture by geostatistical prediction. Geostatistical prediction by the method of kriging requires that we first model the spatially correlated variations of a set of variables and then to use this model to form predictions at unsampled sites (Webster and Oliver, 2007). The predictions have minimized error variance, conditional on the model, and this variance can be reported as a measure of the uncertainty of mapped values. This is a valuable feature of geostatistical prediction, because a rational and robust decision about habitat management at a particular location must be guided not only by the best prediction of the conditions at that location, but also by the uncertainty of those predictions, and the resulting probabilities that other conditions occur.

Geostatistical prediction by kriging is long-established (Matheron, 1963; Journel and Huijbregts, 1978; Webster and Oliver, 2007) and has been applied across the earth and environmental sciences including mining (Costa et al., 2000), hydrology (Zimmermann et al., 2008), soil survey (Burgess and Webster, 1980), regional geochemistry (Rawlins et al, 2003), agronomy (Bishop and Lark, 2007), entomology (Carbajo et al., 2006) and fisheries (Maravelias and Haralabous, 1995). One particular feature of particle size data, not generally encountered in geostatistics, is that they are compositional. That is to say, the percentages of sand, gravel and mud in a given sample sum to 100 by definition, and so these variables are not drawn from an unconstrained three-dimensional sample space but rather are drawn from the constrained simplex space which can be represented as a two-dimensional ternary diagram such as our Figure 1. This has various implications for the statistical properties of the data, and so for their correct analysis (Aitchison, 1986), and this extends to geostatistical analysis and prediction (Pawlowsky-Glahn and Olea, 2004). Lark and Bishop 
(2006) demonstrated the geostatistical modelling and prediction of compositional data on particle size distributions in soil.

In this study our basic data are measurements of the percentages of mud, sand and gravel in the composition of the seabed sediment, but our predictions are of sediment classes. The primary set of classes on which we report here are simplified from the fifteenclass system of Folk (1954) into four broader classes as proposed by Long (2006). These simplified classes are commonly used as the substrate element for habitat mapping and inform at level 3 of the EUNIS classification system (Connor et al., 2006). These classes are shown, projected onto a ternary diagram, in Figure 1. However, because the basic geostatistical modelling is done on the underlying data on the mud, sand and gravel percentages, it is relatively quick to re-map the data according to a modified classification, and we also demonstrate this here.

Our objective in geostatistical mapping according to a legend of classes is to calculate, for any unsampled site, the probability of observing each of the four textural classes there. This enables the data user to identify the most probable class at any location, but also to take account of the probability that other classes occur there. In this paper we use the BGS data set on seabed sediments to generate geostatistical predictions at a denser network of points where direct observations of sediment were not available. We use appropriate transformations of the data to deal with their compositional form, and use the results to compute the probability of occurrence of each of the four texture classes of Long (2006).

\section{Methods}

\subsection{Data collection}

The BGS conducted a systematic programme of regional geological surveys during the 1970s and 1980s (Fannin, 1989) which resulted in the production of a series of 1:250,000

scale maps covering the UK shelf, and a set of offshore regional reports e.g. Cameron et al. (1992). Particle-size data from more than 30000 locations were accumulated during this programme and later project-driven surveys, the locations are shown in Figure 2.

Sediment samples for particle size analysis were recovered from sediment grabs, corers, and dredges. The larger part of the samples were recovered with a Shipek Grab, but analysis was also routinely made on sub-samples from the tops of cores and dredged 
samples. If sufficient material was available the samples were separated into working and archive portions, where between $25 \mathrm{~g}$ and $150 \mathrm{~g}$ of sand (larger samples for more gravelly or muddy sediments) were required for analysis (Balson, 1983). Each sample was analyzed to determine the relative proportions of material in the gravel, sand, and mud particle size classes as defined on the logarithmic Wentworth scale (Wentworth, 1922). The material from each sample was separated into these particle-size classes by both wet and dry sieving on 2-mm and 63- $\mu \mathrm{m}$ sieves. Gravel is classed as the portion which is retained at $2 \mathrm{~mm}$, sand is the portion which passes through $2 \mathrm{~mm}$ but is retained at $63 \mu \mathrm{m}$, and mud is the portion which passes through $63 \mu \mathrm{m}$.

Most samples in the database were acquired prior to the standard use of Global Positioning System (GPS) and Differential GPS (DGPS). Locations for samples collected up to the mid 1980s were based on the Decca Navigator Mainchain and were accurate to approximately $100 \mathrm{~m}$. The Syledis system was then used, with accuracies better than $10 \mathrm{~m}$. The locations of samples collected since the mid 1990s were obtained by GPS and DGPS and are accurate to less than $5 \mathrm{~m}$. The data from the samples held by BGS are stored in an Oracle database and for this study have been projected to UTM zone 30 with reference to the WGS-84 datum.

\subsection{Statistical analysis: overview}

The following sections of this paper provide a complete account of the methodology developed and applied in this paper. This section offers an overview for the reader who does not wish to engage with the detail of the statistics. We have a set of data on the composition of seabed sediments, percentages of sand, gravel and mud. From these we require predictions of the composition at nodes on a dense grid to provide a map. Predictions have uncertainty, and this must be quantified. The first problem (section 2.3) is to transform the data. When analysing data on two or more variables we model their correlations, but the correlations between percentages include artefacts because the percentages must sum to 100 , and so if, for example, the percentage of mud is 80 we know that the percentage of gravel cannot be larger than 20 . We use the additive log-ratio (ALR) transform which converts data on proportions in three size classes (which sum to 1) to values of two new variables. The transform can be inverted to find the percentages in each of the three original classes that 
correspond to any two given values of the transformed variables. We transformed the percentages of gravel, mud and sand to ALR-gravel and ALR-mud; we could choose to transform any two of the three original variables, the effect on our final predictions of the proportions of gravel, mud and sand is the same.

Our next task is to model statistically the spatial variability of the transformed variables. This is explained in section 2.4. We compute what is called a linear model of coregionalization (LMCR). The LMCR for two or more variables allows us to compute the correlation between any two observations of a variable, as a function of the distance in space between them, and similarly to compute the correlation between observations of the different variables that are modelled. We describe in section 2.4 how the LMCR was estimated and validated, including the use of robust estimators that reduce the effects of outlying observations.

When we have an LMCR we can use it to generate statistical predictions of our variables at unsampled locations as functions of the observed values at neighbouring sites. The method is called cokriging. We did this at nodes of a dense grid across the UK Continental Shelf. In addition to the predictions we obtain the variances and covariances of the prediction errors, which quantify the uncertainty about any prediction. This is described in section 2.5. The next step is to obtain predictions of the seabed sediment texture class at the grid nodes (section 2.6). We use a Monte Carlo method to generate a large number of possible values of the true ALR-mud and ALR-gravel at each site, and back-transform each set of values to the original scales of proportions of gravel, mud and sand. The sediment texture class can be identified for each set of values, and the proportion of all the simulated values for a site that belong to a particular class is our estimated probability that that is the true class at the site. We can therefore map the probability of finding each class at our prediction sites, the maximum probability over all the classes and the class of maximum probability. The maximum probability is a measure of how uncertain the prediction is. It takes values from just over $1 / k$, where $k$ is the number of classes, to 1 . If the maximum probability is near the bottom of this scale then the uncertainty of the prediction is large.

We then tested this prediction method by extracting 2000 sites at random, and computing the probability of each class from the remaining data by the cokriging method. In section 2.7 we describe this procedure, and the statistics that we computed to evaluate the 
method. First, we observed the frequency with which the observed class at each validation site belonged to a prediction set of classes defined as either the class of maximum probability, the first- and second-most probable class or the first-, second- or third-most probable class. Second, we compared the values of the maximum probability between the set of sites where the class of maximum probability was the observed class, and the set of sites where it was not. Third, for each class in turn we divided the test sites into groups defined by percentiles of the predicted probability of that class and compared the proportion of sites within each set where the specified class was the observed one with the mean of the predicted probability of that class.

\subsection{Exploratory analysis and data transformation}

A total of 31235 sample sites were available from the seabed sediment database. Sites without complete data on the percent mud, gravel and sand in the sample and sites where the recorded percentages in the mud, gravel and sand classes did not sum to 100 were discarded. Some sites had particle size classes defined on non-standard threshold sizes and these were also discarded. There were also some groups of observations with the same co-ordinates recorded on the UTM-30 projection. Of any set of observations with common co-ordinates just one, randomly selected, observation was retained. After this exploratory editing 28763 observations remained for analysis. Table 1 shows summary statistics for these observations. Note that all the variables are strongly skewed, with coefficients of skewness outside the interval $[-1,1]$.

Each observation in our data set is a composition with $d=3$ components, the percentages of gravel, mud and sand. We represent these by a vector $\mathbf{y}=\left\{y_{1}, \ldots, y_{d}\right\}^{\mathrm{T}}$, where the superscript ' $T$ ' denotes the transpose of a matrix or vector. In the compositional geostatistical analysis proposed by Pawlowsky-Glahn and Olea (2004) these three variables are transformed to their additive log-ratios (ALR):

$$
\mathbf{x}=\left\{\ln \frac{y_{1}}{y_{d}}, \ldots, \ln \frac{y_{d-1}}{y_{d}}\right\} .
$$

Note that the transform replaces a $d$-dimensional variate with a $d$-1-dimensional one. The choice of which variable goes into the denominator of the transformation is arbitrary. Most important is to note that the elements of $\mathbf{x}, x_{1}, \ldots, x_{d-1}$ are now unbounded negative or positive values. The ALR transform is invertible by the additive generalized logistic transform 
$(A G L)$ :

$$
\mathbf{y}=\left\{\frac{\exp \left(x_{1}\right)}{1+\sum_{i=1}^{d-1} \exp \left(x_{i}\right)}, \ldots, \frac{\exp \left(x_{d-1}\right)}{1+\sum_{i=1}^{d-1} \exp \left(x_{i}\right)}, \frac{1}{1+\sum_{i=1}^{d-1} \exp \left(x_{i}\right)}\right\} .
$$

Note that the AGL transform returns proportions that sum to one and must be multiplied by 100 if percentages are required.

The ALR transformation used on compositional variables is undefined if any observed value is zero. Our data contained zero values in all the particle size classes, so an appropriate strategy was needed to deal with these. Martín-Fernandéz and Thió-Henestrosa (2006) discuss the problem that is presented by zero values in compositional data. Although ordinary multivariate statistical methods, which ignore the compositional constraints on the observations, can deal with zero values with no difficulty, they run into problems when the assumptions they entail about the sample space from which the data are drawn do not hold. If we are dealing with a compositional phenomenon which we can reasonably treat as continuous (the composition of seabed sediments around the UK varies spatially, but in a continuous way, not with sharp boundaries at discrete locations) then, where reasonable, the best approach following Martín-Fernandéz and Thió-Henestrosa (2006) is to assume that zero values arise from rounding error, and can be assumed to represent very small non-zero values not resolved by measurement.

The smallest non-zero value recorded for any size fraction was 0.01 percent. After discussion with the data managers it seemed reasonable to assume that the zero values arise from rounding error, and that the true value corresponding to an recorded value of zero is somewhere in the interval $(0,0.01)$. A method of imputation is needed to replace zero values with a non-zero value. We used the simple replacement method discussed by MartínFernandéz and Thió-Henestrosa (2006). Let $\mathbf{y}=\left\{y_{1}, \ldots, y_{d}\right\}^{\mathrm{T}}$ be a single observation of a compositional variate, percentages summing to 100. Each of these values $y_{i}$, where $i$ represents any component of the composition, is replaced by a new value $y_{i}^{\prime}$ where

$$
\begin{aligned}
y_{i}^{\prime} & =y_{i} \text { if } y>0, \\
& =y_{i}+\delta_{r} \text { if } y=0,
\end{aligned}
$$

where $\delta_{r}$ is some small value. The values are then renormalized to give a new composition 
$\mathbf{z}=\left\{z_{1}, \ldots, z_{d}\right\}$ with no zero values, and with terms summing to 100

$$
z_{i}=100 \times \frac{y_{i}^{\prime}}{\sum_{j=1}^{d} y_{j}^{\prime}}
$$

An obvious choice of $\delta_{r}$ in our case is 0.005 , half the smallest non-zero value in the data, 0.01 which we treat as a limit of resolution for our measurements. Following the recommendation of Martín-Fernandéz and Thió-Henestrosa (2006) we examined the effect of using a range of values, $0<\delta_{r}<0.01$ on basic statistics of the modified values after transformation to additive log-ratios, with the sand content used as the denominator variable. Figure 3 shows the coefficient of variation (the ratio of the standard deviation to the absolute value of the mean) for the ALR transform of mud and gravel content, and the correlation between these values with different values of $\delta_{r}$. The sensitivity is small, so $\delta_{r}=0.005$ was used.

Table 1 shows summary statistics for the ALR-transformed mud and gravel content. Note that the transformed variables are much more symmetrically distributed than the original ones, with small coefficients of skew.

\subsection{Variograms, cross-variograms and the linear model of coregionalization}

After the ALR transformation of our data we have two values, $z_{\mathrm{m}}$ and $z_{\mathrm{g}}$, the transformed content of mud and gravel respectively, at any sampled location s. It is beyond the scope of the present paper to give a full discussion of the geostatistical model, and we refer the reader to the books by Webster and Oliver (2007) and Pawlowsky-Glahn and Olea (2004) for more detail. To summarize, in geostatistics we treat these values as realizations of two random variables, $Z_{\mathrm{m}}(\mathrm{s})$ and $Z_{\mathrm{g}}(\mathrm{s})$ respectively. Under the assumption of weak stationarity it is assumed that the variables $Z_{\mathrm{m}}(\mathbf{s})$ and $Z_{\mathrm{g}}(\mathbf{s})$ fluctuate about constant mean values, $\mu_{\mathrm{m}}$ and $\mu_{\mathrm{g}}$. Further, it is assumed that observations of these variables at any two locations, $\mathbf{s}$ and $\mathbf{s}^{\prime}$, have a covariance which can be expressed as a function simply of the lag interval $\mathbf{s}-\mathbf{s}^{\prime}$. Thus:

$$
\begin{aligned}
\operatorname{Cov}\left[Z_{\mathrm{m}}(\mathbf{s}), Z_{\mathrm{m}}\left(\mathbf{s}^{\prime}\right)\right] & =\mathrm{E}\left[\left\{Z_{\mathrm{m}}(\mathbf{s})-\mu_{\mathrm{m}}\right\}\left\{Z_{\mathrm{m}}\left(\mathbf{s}^{\prime}\right)-\mu_{\mathrm{m}}\right\}\right] \\
& =C_{\mathrm{m}}\left(\mathbf{s}-\mathbf{s}^{\prime}\right)
\end{aligned}
$$

where $\operatorname{Cov}[$.$] and \mathrm{E}[$.$] denote, respectively, the covariance and the statistical expectation$ of the terms in brackets, and $C($.$) denotes a covariance function. The covariance of Z_{\mathrm{g}}(\mathbf{s})$ 
could similarly be defined. The covariance function expresses the spatial dependence of the variation of some property. It declines to zero at some separation (the range of the process), but joint observations of the variable at locations closer together than the range are spatially correlated. We are also interested in the joint variation of $Z_{\mathrm{m}}(\mathbf{s})$ and $Z_{\mathrm{g}}(\mathbf{s})$ and its spatial dependence. This is expressed by the cross-covariance function

$$
C_{\mathrm{m}, \mathrm{g}}(\mathbf{h})=\mathrm{E}\left[\left\{Z_{\mathrm{m}}(\mathbf{s})-\mu_{\mathrm{m}}\right\}\left\{Z_{\mathrm{g}}(\mathbf{s}+\mathbf{h})-\mu_{\mathrm{g}}\right\}\right]
$$

Note that, in general, $C_{\mathrm{m}, \mathrm{g}}(\mathbf{h}) \neq C_{\mathrm{m}, \mathrm{g}}(-\mathbf{h})$.

The cross-covariance function is not necessarily symmetrical. However, it is common to assume symmetry, unless there is strong evidence to the contrary (Webster and Oliver, 2007), because this allows us to use the linear model of coregionalization, described below, to model the joint spatial variation of two or more variables for their joint spatial prediction by kriging (Journel and Huijbregts, 1978). We may also weaken the assumption of second order stationarity to the intrinsic hypothesis of stationarity. Under this assumption:

$$
\mathrm{E}\left[\left\{Z_{\mathrm{m}}(\mathbf{s})-Z_{\mathrm{m}}(\mathbf{s}+\mathbf{h})\right\}^{2}\right]=2 \gamma_{\mathrm{m}}(\mathbf{h}), \forall \mathbf{s}
$$

where $\gamma_{\mathrm{m}}(\mathbf{h})$ the variogram function depends only on the lag vector $\mathbf{h}$. Again, a variogram function is defined similarly for $Z_{\mathrm{g}}$. Under the assumption of symmetry we can express the joint spatial variation of our variables by the cross variogram:

$$
\gamma_{\mathrm{m}, \mathrm{g}}(\mathbf{h})=\frac{1}{2} \mathrm{E}\left[\left\{Z_{\mathrm{m}}(\mathbf{s})-Z_{\mathrm{m}}(\mathbf{s}+\mathbf{h})\right\}\left\{Z_{\mathrm{g}}(\mathbf{s})-Z_{\mathrm{g}}(\mathbf{s}+\mathbf{h})\right\}\right]
$$

Webster and Oliver (2007) describe how estimates of the variograms and cross-variogram can be made from data. For any pair of observation sites, $\mathbf{s}_{i}, \mathbf{s}_{j}$ the lag-vector $\mathbf{s}_{i}-\mathbf{s}_{j}$ is assigned to a lag bin centred on $\mathbf{h}$ if $\mathbf{s}_{i}-\mathbf{s}_{j} \approx \mathbf{h}$ to some degree of approximation. We denote the number of pairs of observations in the lag bin by $N_{\mathbf{h}}$. For notational convenience we write the pair difference as a new variable:

$$
w_{\mathrm{m}, i, j}=z_{\mathrm{m}}\left(\mathbf{s}_{i}\right)-z_{\mathrm{m}}\left(\mathbf{s}_{j}\right),
$$

and put the $N_{\mathbf{h}}$ pair comparisons $w_{\mathrm{m}, i, j}, \forall \mathbf{s}_{i}-\mathbf{s}_{j} \approx \mathbf{h}$ into the vector $\mathbf{w}_{\mathrm{m}}(\mathbf{h})$. We can then write the standard variogram and cross-variogram estimators as:

$$
\widehat{\gamma}_{\mathrm{m}}(\mathbf{h})=\frac{1}{2 N_{\mathbf{h}}} \mathbf{w}_{\mathrm{m}}^{\mathrm{T}}(\mathbf{h}) \mathbf{w}_{\mathrm{m}}(\mathbf{h}),
$$


and

$$
\widehat{\gamma}_{\mathrm{m}, \mathrm{g}}(\mathbf{h})=\frac{1}{2 N_{\mathbf{h}}} \mathbf{w}_{\mathrm{m}}^{\mathrm{T}}(\mathbf{h}) \mathbf{w}_{\mathrm{g}}(\mathbf{h})
$$

The definition of a lag bin may be simplified further if we can assume that the variogram is isotropic and so depends only on the lag distance, $|\mathbf{s}|$ and not the direction.

It is well-known (e.g. Cressie and Hawkins, 1980) that these standard variogram and cross-variogram estimators are susceptible to the effects of outlying observations on the data, be these marginal outliers (observations that appear unusual by comparison to the overall distribution of observations) or spatial outliers (observations that are unusual compared to their neighbours). Lark (2003) presented two robust estimators for the crossvariogram, which reduce the effects of outlying observations on the estimates. In this paper we used one of these estimators, adapted for our large data set. The estimator requires a robust estimator of the standard deviation applied to a vector of $n$ observations, $\mathbf{v}$, and in this study we used the median absolute deviation from the median:

$$
\operatorname{MAD}(\mathbf{v})=1.4826 \times \operatorname{median}\left(\left\{\left|v_{1}-\operatorname{median}(\mathbf{v})\right|, \ldots\left|v_{n}-\operatorname{median}(\mathbf{v})\right|\right\}^{\mathrm{T}}\right)
$$

where median() denotes the median value of the elements of the vector in the brackets. Following Ma and Genton (1998), we can define a robust estimator of the covariance of two variables with observations in vectors $\mathbf{v}$ and $\mathbf{u}$ by:

$\widehat{C}_{\mathrm{R}}(\mathbf{u}, \mathbf{v})=\frac{\operatorname{MAD}(\mathbf{u}) \operatorname{MAD}(\mathbf{v})}{4}\left\{\operatorname{MAD}^{2}\left(\frac{\mathbf{u}}{\operatorname{MAD}(\mathbf{u})}+\frac{\mathbf{v}}{\operatorname{MAD}(\mathbf{v})}\right)-\operatorname{MAD}^{2}\left(\frac{\mathbf{u}}{\operatorname{MAD}(\mathbf{u})}-\frac{\mathbf{v}}{\operatorname{MAD}(\mathbf{v})}\right)\right\}$

Following Lark (2003) we can then obtain a robust estimator of the cross-variogram by

$$
\widehat{\gamma}_{\mathrm{m}, \mathrm{g}}^{\mathrm{R}}(\mathbf{h})=\frac{1}{2} \widehat{C}_{\mathrm{R}}\left(\mathbf{w}_{\mathrm{m}}, \mathbf{w}_{\mathrm{g}}\right)
$$

The corresponding variogram estimator:

$$
\widehat{\gamma}_{\mathrm{m}}^{\mathrm{R}}(\mathbf{h})=\frac{1}{2} \widehat{C}_{\mathrm{R}}\left(\mathbf{w}_{\mathrm{m}}, \mathbf{w}_{\mathrm{m}}\right)
$$

is equivalent to the robust estimator proposed by Dowd (1984). In practice we select between robust estimators of variograms and the standard estimator by a cross-validation procedure, which is described below.

The empirical cross-covariances of the ALR-transformed mud and gravel content did not indicate marked asymmetry. Asymmetry might be expected in a study of a relatively 
small region with pronounced trends in composition due to currents, but in a large-scale study many such local trends are averaged. There was no evidence for anisotropy in the empirical variograms (dependence on the direction as well as the lag distance) so lag bins were defined with respect to distance only.

In order to do kriging we require continuous functions of lag distance for the variograms and cross-variograms. These are obtained by fitting appropriate models to the estimates for different lag distances. We cannot use any function for this purpose, but only 'authorized' functions. This is necessary because the variogram function can be used to compute the variance of some linear function of a random variable, and using an authorized function is a sufficient condition to ensure that this variance is not negative. One such authorized function is the spherical function:

$$
\begin{aligned}
\operatorname{Sph}(h \mid a) & =\left\{\frac{3 h}{2 a}-\frac{1}{2}\left(\frac{h}{a}\right)^{3}\right\}, \quad h \leq a \\
& =1, \quad h>a
\end{aligned}
$$

where $a$ is a parameter of the function, the range. The function increases from 0 to 1 as $h$ increases from 0 to $a$. A typical variogram model may take the form

$$
\begin{aligned}
\gamma(h) & =c_{0}+c_{1} \operatorname{Sph}\left(h \mid a_{1}\right)+c_{2} \operatorname{Sph}\left(h \mid a_{2}\right) \quad h>0 \\
& =0, \quad h=0 .
\end{aligned}
$$

This model requires some explanation. First, it is a discontinuous function, equal to zero at lag 0, but approaching a non-zero intercept, $c_{0}$ as $h$ goes to zero. This is the 'nugget' effect in geostatistics. The variance, $c_{0}$, is a component of the overall variance of the random variable, and arises from sources of variation that show no spatial dependence over lag distances larger than or equal to the finest interval between observations in our data. Its spatial structure is therefore not resolved. Note that this may include measurement error. As $h$ increases so the variogram increases to a maximum value of $c_{0}+c_{1}+c_{2}$. This is called the sill variance. Two observations separated by a distance greater than $a_{2}$ are spatially independent of each other, but two observations separated by a shorter distance are likely to be more similar, hence the smaller value of the variogram. The two components with spatial dependence at lags up to $a_{1}$ and $a_{2}$ respectively are modelled as independent additive components of the variable. One shows spatial dependence at rather shorter distances than 
the other. In some circumstances just one spatially dependent component of the variogram model is needed, but any number of nested components can be used. This flexibility means that the variogram model can represent variables in which the variation is driven by disparate processes at very different spatial scales.

For co-kriging it is necessary to model jointly the variograms and cross-variograms of the variables. The model for our case with two variables can be expressed as a $2 \times 2$ matrix

$$
\boldsymbol{\Gamma}(h)=\left[\begin{array}{cc}
\gamma_{\mathrm{m}}(h) & \gamma_{\mathrm{m}, \mathrm{g}}(h) \\
\gamma_{\mathrm{m}, \mathrm{g}}(h) & \gamma_{\mathrm{g}}(h)
\end{array}\right]=\mathbf{b}_{0}+g_{1}(h) \mathbf{b}_{1}+g_{2}(h) \mathbf{b}_{2},
$$

where the functions $g_{1}(h), g_{2}(h)$ are authorized variogram functions and the matrices $\mathbf{b}_{0}, \mathbf{b}_{1}, \ldots$ are covariance matrices for the components of variation represented by the variogram functions which provide their scalar multipliers. Note, therefore, that $b_{0}$ is a covariance matrix of nugget components. This is the linear model of coregionalization (LMCR) (Journel and Huijbregts, 1978) which is the standard way to model joint spatial dependence, ensuring validity.

Estimates of the variograms and cross-variogram of ALR-transformed gravel and mud content were obtained by the standard estimators, Eq[10] and Eq[11] above, and the robust alternatives, Eq[14] and Eq[15]. An LMCR was fitted to each set of estimates using the simulated annealing algorithm presented by Lark and Papritz (2003). The two sets of variogram models for ALR-transformed mud and gravel content were then used in a cross-validation. Each datum in turn was excluded from the data set and predicted by ordinary kriging from the remaining observations. The corresponding kriging error at a site could then be calculated as the difference between the prediction and the known value. Furthermore, the kriging variance, the expected mean-squared error of the kriging prediction, was computed for each prediction. The standardized squared kriging error was calculated by dividing the square of the kriging error by the kriging variance. If the kriging variances give a good account of the uncertainty, then the mean of this statistic over all observations should be close to 1.0. However, Lark (2000) showed that the average squared standardized kriging error over validation points is a poor diagnostic in the presence of outliers since these can affect both the observed kriging error and the kriging variance (via the variogram). The median standardized squared kriging error is a better diagnostic, (Lark, 2000) and should be close to 0.455 if the distribution of kriging errors can be assumed to be normal. On this basis a choice was made between the LMCR based on the standard estimator and the robust alter- 
native for further use in cokriging. Standardized kriging errors were also calculated from the cross validation output, this is the prediction error divided by the square root of the kriging variance.

\subsection{Cokriging and back-transformation}

A detailed account of ordinary co-kriging is presented by Webster and Oliver (2007) and by Pawlowsky-Glahn and Olea (2004). In summary, the ordinary co-kriging estimate of $Z_{\mathrm{g}}\left(\mathbf{s}_{0}\right)$ at some location, $\mathbf{s}_{0}$, denoted $\tilde{Z}_{\mathrm{g}}\left(\mathbf{s}_{0}\right)$ is given by a weighted average of the observed values of the primary variable, $z_{\mathrm{g}}$ and any secondary variables, in our case $z_{\mathrm{m}}$, at locations $\mathbf{s}_{1}, \mathbf{s}_{2}, \ldots, \mathbf{s}_{N}$ in some set of $N$ neighbouring sample sites

$$
\tilde{Z}_{\mathrm{g}}\left(\mathbf{s}_{0}\right)=\sum_{i=1}^{N} \lambda_{\mathrm{g}, i}^{\mathrm{g}} z_{\mathrm{g}}\left(\mathbf{s}_{i}\right)+\sum_{i=1}^{N} \lambda_{\mathrm{m}, i}^{\mathrm{g}} z_{\mathrm{m}}\left(\mathbf{s}_{i}\right),
$$

where the values of $\lambda_{\mathrm{g}, i}^{\mathrm{g}}$ and $\lambda_{\mathrm{m}, i}^{\mathrm{g}}$ are the ordinary cokriging weights, the superscript, $\mathrm{g}$, in each case is an index not a power, indicating that the weight is for the prediction of $Z_{\mathrm{g}}$, and the subscript $\mathrm{g}$ or $\mathrm{m}$ indicates whether the weight is applied to observed values of the transformed gravel or mud content respectively. The values of these weights are found that will minimize the expected squared error of the cokriging estimate:

$$
\sigma_{\mathrm{g}}^{2}\left(\mathbf{s}_{0}\right)=\mathrm{E}\left[\left\{\tilde{Z}_{\mathrm{g}}\left(\mathbf{s}_{0}\right)-Z_{\mathrm{g}}\left(\mathbf{s}_{0}\right)\right\}\right]
$$

subject to the constraint that $\sum_{i=1}^{N} \lambda_{\mathrm{g}, i}^{\mathrm{g}}=1$ and $\sum_{i=1}^{N} \lambda_{\mathrm{g}}^{\mathrm{g}}=0$. The weights are found by solution of the ordinary cokriging equations, which can be set up in terms of the values of the variograms and cross-variograms for the lag intervals between the $N$ neighbouring sites, and between these sites and the target site $\mathbf{s}_{0}$. Along with the cokriging prediction we also obtain a calculated value of the cokriging variance, defined in Eq (20). The cokriging prediction can also be obtained for $\tilde{Z}_{\mathrm{m}}\left(\mathrm{s}_{0}\right)$ with $Z_{\mathrm{m}}$ now the secondary variable. The full covariance matrix of cokriging prediction errors for both sets of variables, $\mathbf{C}_{x}\left(\mathbf{s}_{0}\right)$ is also obtained. Element $\{i, i\}$ of this matrix contains the variance of the cokriging errors for the $i$ th variable, and element $\{i, j\}$ contains the covariance of the cokriging errors for the $i$ th and $j$ th variable, see Pawlowksky-Glahn and Olea (2004).

We used ordinary cokriging, as encoded in the COКВ3DМ program of Deutsch and Journel (1992), to compute predictions of $Z_{\mathrm{g}}$ and $Z_{\mathrm{m}}$, the ALR-transformed percentages of gravel and mud at a set of target points corresponding to the nodes of the BGS's bathy- 
metric data set Digibath. All the 28763 observations available after the exploratory analysis described in section 2.3 were used for cokriging. At each location the covariance matrix of cokriging errors, $\mathbf{C}_{x}$ was also calculated and recorded.

At any location, $\mathbf{s}_{0}$, where cokriging estimates of the ALR-transformed percentages of gravel and mud have been computed it is possible to back-transform these estimates to the original scale of measurement by applying the AGL transform, Eq. [2], and then multiplying by 100 to convert proportions to percentages. However, while the cokriged estimates are best linear unbiased predictions, because the mean square error is minimized by kriging, the back-transformed values are not the best linear unbiased predictions on the original scale of measurement. Pawlowsky-Glahn and Olea (2004) report that an unbiased backtransformation is not available for kriged predictions of ALR-transformed variables. This is not a concern here since our objective is to predict the seabed texture class according to the classification of in Figure 1a, and to quantify its uncertainty.

\subsection{Computing the local probability of each texture class}

$Z_{\mathrm{g}}\left(\mathbf{s}_{0}\right)$ and $Z_{\mathrm{m}}\left(\mathbf{s}_{0}\right)$ are random variables with a joint probability distribution. The cokriged estimates, $\tilde{Z}_{\mathrm{g}}\left(\mathbf{s}_{0}\right)$ and $\tilde{Z}_{\mathrm{m}}\left(\mathbf{s}_{0}\right)$ respectively, are estimates of the mean value of these random variables, conditional on the neighbouring observations from which they have been computed. The covariance matrix $\mathbf{C}_{x}\left(\mathbf{s}_{0}\right)$ gives the variances and covariances of the distribution. If it is reasonable to assume that the prediction errors have a bivariate normal distribution, then these statistics wholly characterize the conditional joint distribution of $Z_{\mathrm{g}}\left(\mathrm{s}_{0}\right)$ and $Z_{\mathrm{m}}\left(\mathbf{s}_{0}\right)$. This provides us with an approach to estimate the probability that the sediment at location $\mathbf{s}_{0}$ belongs to any particular class in Figure 1a by Monte Carlo integration of the joint probability density function over each class. We did this as follows.

Because the covariance matrix $\mathbf{C}_{x}\left(\mathbf{s}_{0}\right)$ is necessarily symmetrical and positive-definite, it can be factorized by a Lower-Upper decomposition:

$$
\mathbf{C}_{x}\left(\mathbf{s}_{0}\right)=\mathbf{L L}^{\mathrm{T}}
$$

If the vector $\mathrm{g}$ contains two independent normal random variables with mean zero and variance 1.0, then $\mathbf{w}=\mathbf{L g}$ is a vector containing two random variables drawn from the normal distribution with mean zero and covariance matrix $\mathbf{C}_{x}\left(\mathbf{s}_{0}\right)$ and

$$
\left[\tilde{Z}_{\mathrm{g}}\left(\mathbf{s}_{0}\right), \tilde{Z}_{\mathrm{m}}\left(\mathbf{s}_{0}\right)\right]^{\mathrm{T}}+\mathbf{w}
$$


is a random realization from the conditional joint distribution of $Z_{\mathrm{g}}\left(\mathbf{s}_{0}\right)$ and $Z_{\mathrm{m}}\left(\mathbf{s}_{0}\right)$. These values can be back-transformed to the corresponding proportions of gravel, mud and sand by the AGL transform. We used the RNMVN subroutine in the IMSL numerical library to generate 5000 independent realizations of $Z_{\mathrm{g}}\left(\mathbf{s}_{0}\right)$ and $Z_{\mathrm{m}}\left(\mathbf{s}_{0}\right)$ for each location where kriging predictions had been obtained. Each realization was back-transformed to percent gravel, mud and sand, and the corresponding texture class from Figure 1a was identified. At each location the proportion of simulated values falling in each of the four classes was treated as an estimate of the conditional probability of each of those classes' occurring at the site.

\subsection{Validation by jacknifing}

As a test of this procedure a set of 2000 sites from the seabed sediment data were selected independently and at random and removed from the main data set. The ordinary kriging predictions, $\tilde{Z}_{\mathrm{g}}\left(\mathbf{s}_{0}\right)$ and $\tilde{Z}_{\mathrm{m}}\left(\mathbf{s}_{0}\right)$, and their covariance matrix, $\mathbf{C}_{x}\left(\mathbf{s}_{0}\right)$, were obtained at each site, using the remaining data, and the procedure described in section 2.5 was used to compute the conditional probability of the occurrence of each class at each site. These results were then assessed in three ways.

First, we noted the frequency over all validation sites with which the recorded sediment texture class corresponded to each of three prediction sets of classes. By a prediction set we mean simply a set of one or more classes offered as predictions of the class at a site, and the observed class is held to correspond to the prediction set if it is one member of the set. The first prediction set was simply the class with the largest probability at the site estimated as described in section 2.5. The second set comprised the class with the largest probability and that class with the second-largest probability. The third set comprised all but the class with the smallest probability. Clearly as the number of classes in the prediction set increases then the probability that the observed class belongs to the set is expected to increase, but the practical value of the prediction diminishes. To assess the value of the information provided by the geostatistical procedure we also computed the frequency with which the observed class corresponded to prediction sets which are the same at all locations, and are based on the overall proportions of the different classes in the validation set - i.e. at all cases the most probable class is the most frequent one (Sand and Muddy Sand), the second-most probable class is the second-most frequent overall (Coarse) and the 
third-most probable class is Mud and Sandy Mud. These results indicate the confidence that we can place in predicting that the class at a location is the class with maximum probability, and how often we will be correct if our prediction is that the class is either the most probable or the second-most probable.

Second, we evaluated the probability of the class of maximum probability at a site as a measure of uncertainty. To do this we divided the observations into those for which the class of maximum probability corresponded to the observed class, and those where it did not. We then compared the summary statistics for the maximum probability between these two groups.

Finally, we attempted a direct assessment of the locally computed probabilities for all three classes. For each class in turn the sites were then divided into five groups by the quintiles (20th, 40th, 60th and 80th percentiles) of the conditional probability of finding the class under consideration. The average value of the conditional probability within each group was computed. This can be treated as an approximation to the probability of an observation in that group belonging to the class in question. The proportion of the sites in that group at which the class under consideration actually occurred was then noted. A plot of the proportion of sites with the group against the average probability, for over all five groups was made for each class. If the computed probabilities are reliable then these should fall near the bisector.

\section{Results}

Table 2 shows the cross-validation results for ordinary kriging of ALR-transformed mud and gravel using the variogram models from the LMCRs fitted, respectively, to the standard and robust estimates. Note that in all cases the mean kriging error was close to zero. The median value of the standardized squared kriging error was closest to the expected value for normal kriging errors $(0.455)$ when the robust estimator was used. The mean standardized squared kriging error was rather larger than 1.0 for the robust estimator, this is expected because outliers inflate the observed squared kriging errors in their vicinity, but do not inflate the kriging variances based on a robustly estimated variogram (Lark, 2000). For this reason the robust estimator was selected. Figure 4 shows the robust point estimates of the variograms and cross-variogram for ALR-transformed gravel and mud content, and the fitted 
functions from the LMCR, the parameters of the fitted LMCR are presented in Table 3. A double-spherical variogram model with three nested components was selected among the standard models used for this purpose on the basis of the Akaike information criterion as a measure of model fit (Webster and Oliver, 2007). The covariance components in the matrices $\mathbf{b}_{0}, \mathbf{b}_{1}$ and $\mathbf{b}_{2}$ are also rescaled to correlations (structural correlations). These express the strength of the linear relationship between the three modelled components of the spatial variation of ALR-transformed gravel and mud content. Note the scale-dependence of this relationship. There is a positive correlation between the components of the two variables that are not spatially correlated at the scales resolved by the data set. There is a weaker, but still positive, correlation between the components that are correlated up to about $4 \mathrm{~km}$, and a weak negative correlation between the components at the coarsest spatial scale, correlated up to about $18 \mathrm{~km}$.

Figure 5 shows the empirical probability density function (pdf) of the standardized kriging errors for the two variables, estimated by a kernel estimator using the KERNELDENSITY procedure in GenStat (Payne, 2010) and the method of Sheather and Jones (1991) to select the kernel bandwidth. A standard normal pdf is superimposed. This shows that the distribution of the kriging errors appears approximately normal, but with more observations than would be expected in the tails of the distribution, probably reflecting the effects of outliers in the cross-validation procedure. This result gives us confidence in the assumption of normality made when computing the conditional probabilities for the texture classes from the cokriging output as described in section 2.6.

Figure 6a-d shows the local conditional probability for each of the texture classes, and Figure 6e shows the local class with maximum probability. Note that the class of Mixed sediment is sparse and not distributed in large patches, so the sites at which it is the class of maximum probability are not evident on a map at this scale. Figure 7 shows the value of the maximum probability at each location.

In Figure 8 is plotted the probability that the observed class at each site is included in increasingly broad prediction sets, formed by including classes sequentially in reverse order of probability, estimated from the co-kriging results. Also plotted are the probabilities of inclusion when the prediction sets are defined by the overall frequencies of the classes in the validation set. Note that the probability that the class with maximum probability obtained from 
the kriging is the class observed at the validation site is 0.71 , and that the probability that the observed class is the class of maximum probability or the second-most probable class is 0.92 . The corresponding values when the prediction sets are determined by the overall probabilities are 0.46 and 0.77 respectively. These results indicate the additional information that the cokriging procedure provides on the expected texture class at an unsampled site. Note that the probability that the class at a site is either the most probable or the second most probable as determined by cokriging is large (0.92), and larger than the probability that the class at a site is either the most frequent or second most frequent class overall $(0.77)$.

Table 4 provides summary statistics for the computed probability of the class of maximum probability at sites divided into those where the class of maximum probability was the one observed, and those where some other class was observed. Note that the median value of the maximum probability among the correctly allocated classes is 0.64 , which is equal to the third quartile among the incorrectly allocated classes. This suggests that the maximum probability may be an informative indicator of local uncertainty. A threshold value to distinguish between the sites where the class is correctly predicted by the class of maximum probability and those where it is not, corresponding to the discriminant function is 0.6. Note that the proportion of correctly allocated sites among those with a maximum probability greater than or equal to 0.6 is 0.82 , and among those with a smaller maximum probability it is 0.6 . This suggests that we might treat the class of maximum probability as the predicted class at a site with some confidence where the maximum probability exceeds 0.6 , but at sites where it is smaller we may wish take greater account of the uncertainty, perhaps examining the implications for any management decision we make of the true class being the one with the second-largest probability.

In Figure 9 are plotted, for each class, the observed proportions of sites that belong to the class within the quintile groups of the predicted probabilities of that class. This is plotted against the average value of that probability in each group. For the two largest classes the observed proportions and the average probabilities are strongly associated with the observations close to the bisector. There is a tendency for the average probabilities somewhat to under-predict the observed proportions where these values are large, and over-predict them where they are small. For the two less-frequent classes the tendency for the average probability of the class to over-predict the observed proportions where these 
are smaller is more pronounced.

In Figure 10 is plotted the class of maximum probability and the probability of the class of maximum probability for the alternative seabed sediment texture class proposed by James et al. (2010) and illustrated in Figure 1b.

\section{Discussion}

The geostatistical analysis of the sediment data shows that there is complex multiscale variability in the composition of seabed sediments. Variation was found at three nested scales. There is significant variation at the coarsest scale, with spatial dependence up to about $18 \mathrm{~km}$. It is interesting to note that the correlation between the mud and gravel content (as transformed to log ratios) is weak (and slightly negative) at this coarsest scale. There is also variation at a rather finer scale, spatially dependent up to about $4 \mathrm{~km}$. The variation of the mud and gravel components is rather more strongly and positively correlated at this scale. The strongest correlation is seen in the third component, which is variation which is not spatially correlated at scales resolved by the sampling scheme that gives rise to our data. This suggests that different processes are causing the variation in sediment composition at the contrasting scales. Further work to estimate and map separately the nested components of variation by factorial cokriging may help to elucidate those processes.

The complex multiscale processes that determine the composition of seabed sediments give rise to the pattern of variation represented by the map of the most probable classes, shown in Figure 6e. A map to the same legend classes was produced for the UKSeamap project (Figure 11), which incorporated physical and biological data to produce a habit map for the UK marine area according to the EUNIS classification system (Connor et al., 2006; McBreen et al., 2010). This seabed sediment layer, as part of UKSeamap, facilitates habitat mapping at level 3 of the EUNIS classification, and was produced primarily by modifying the existing BGS seabed sediment map of the UK (Cooper et al., 2010) which is based on the classes of Folk (1954). The map in Figure 6e is consistent with the

UKSeamap layer in Figure 11. Note that the 'rock' layer within UKSeamap (Figure 11) is defined where rock is present within $0.5 \mathrm{~m}$ of the seabed, therefore this class incorporates an element of sub-seabed interpretation and is more widespread than in Figure 6e where 'rock' is recorded where this is the substrate indicated in the DigiBath data. The advantages 
of the geostatistical map are as follows.

First, the geostatistical mapping procedure generates directly an indication of the uncertainty in the predictions. This study has shown that the uncertainty measures arising from the geostatistical model are reliable. The representation of uncertainty in the map (e.g. Figure 7) shows that a map based on the most probable local class is not uniformly accurate. Consider, for example, the English Channel which is almost all allocated to 'Coarse' with the exception of the waters of the south-west coast. If one examines the map for probability of class Coarse (Figure 6a), then it is apparent that our uncertainty about this allocation is greater in the West than is is around the Isle of Wight. Similarly the North Sea is largely in 'Sand and Muddy Sand' but Fig 6d shows substantial variations in the confidence of this allocation. Comparable uncertainty will underly maps produced by other methodologies, the advantage of the geostatistical approach is that the uncertainty is minimized in that the cokriging predictions of the transformed proportions of the different size classes are the best linear unbiased predictions, and the uncertainty is quantified directly as part of the modelling procedure.

Second, the geostatistical mapping procedure is based on a spatial model and cokriging estimates of the basic particle size fractions, and the results of these initial steps can be rapidly processed to generate maps according to different classifications on the Ternary diagram. This is illustrated by Figure 9 which is based on a quite different classification to that of Long (1996); the extent of the class 'Sand' according to the classification of James et al. (2001) is much more extensive than the class named 'Sand' in the scheme of Long (1996). Note that the probability of the class of maximum probability in Figure 9(b) shows that this map has rather greater uncertainty attached to its predictions, particularly near the coast. This is because the class Mixed in the classification of James et al. (2010) tends to occur in fine-scale assemblages with other classes, it only occurs as the class of maximum probability over a restricted region, but its probability of occurring is quite large over much of the mapped area. This suggests that scientists, when considering which classification to use, should consider not only its inherent properties (e.g. for representing the effects of substrate on ecological communities), but also the confidence with which it can be mapped from available data.

The geostatistical maps of sediment classes are based only on the particle size data 
and the spatial model. The conventional maps of seabed sediment made by geologists use other data (geophysical measurements) and geological interpretation. However, it would be possible to incorporate these other sources of information into the geostatistical approach. This is because the geostatistical model is a particular case of the linear mixed model (Lark et al, 2006). The variograms describe spatially correlated random variables in the model, but the model can also include fixed effects, such as a continuous geophysical covariate which is correlated with the target property (e.g. the gravel content of the sediment) or a categorical variable, such as a set of geological units delineated by boundaries. The relationship between the fixed effects and the target variable is exploited to improve prediction, and the cokriging component of prediction exploits the spatial correlation of the variation in the target properties that remains unexplained. The potential to combine geostatistical prediction and these additional sources of information is a topic for further investigation.

In this study we discarded data with particle-size classes defined on non-standard thresholds. In some circumstances, particularly in international areas where data have been collected by different organizations, it may be necessary to work with such data. The recently published method of Weltje and Robinson (2012) could be used to this end.

The jacknifing exercise to validate the geostatistical predictions and the measures of uncertainty showed that the overall 'purity' of the legend units in Figure $6 e$ is about 0.7 , i.e. the probability that the class of maximum probability is the observed class at a randomly located site is 0.7 . This figure is quite respectable (at least by comparison to other types of spatial information in the geosciences, such as soil maps), but it might surprise the average policy or regulatory 'consumer' of spatial information. Overall the probability that the observed class is the most probable or second-most probable is 0.92 .

The jacknifing also showed that the probability of the class of maximum probability is a useful measure of uncertainty. When this is less than 0.6 then the probability that the class of maximum probability is the observed class is just 0.6 (0.82 otherwise). This probability (Fig 7) may therefore be used as a guide to interpreting the predictions, where it is small we may wish to account for the possibility that the second-most probable class occurs at a site in any decision making. Fig 7 also shows where the uncertainty is greatest, for example of the South-West and coastal waters up the East Coast. 


\section{Conclusions}

To conclude, geostatistical prediction of the composition of seabed sediment texture, based on additive log-ratio transforms and cokriging can be used to produce maps of the probability that different texture classes are found at locations around the UK continental shelf. This method produces results consistent with conventional mapping, but the semiautomated nature of statistical mapping means that maps to alternative classifications can be produced rapidly. Furthermore, statistical mapping generates measures of uncertainty in the final map, which have been shown in this paper to be reliable. There is scope to develop the methodology in this paper further. In particular, it may be fruitful to attempt to combine geostatistical prediction with conventionally-mapped geological boundaries and geophysical data. 


\section{References}

Aitchison, J. 1986. The Statistical Analysis of Compositional Data. Chapman and Hall, London.

Balson, P.S. 1983. Method of routine analysis of sediment samples in the marine geology unit. Institute of Geological Sciences, Continental Shelf Division. Institute of Geological Sciences, Nottingham.

Bishop, T.F.A., Lark, R.M. (2007) A landscape-scale experiment on the changes in available potassium over a winter wheat cropping season. Geoderma 141, 384-396.

Burgess, T.M., Webster, R. 1980. Optimal interpolation and isarithmic mapping of soil properties. I. The semivariogram and punctual kriging. Journal of Soil Science, 31, 315-331.

Cameron, A., Askew, N. (eds.). 2011. EUSeaMap - Preparatory Action for development and assessment of a European broad-scale seabed habitat map final report. Available at http://jncc.gov.uk/euseamap

Cameron, T.D.J., Crosby, A., Balson, P.S., Jeffery, D.H., Lott, G.K., Bulat J., Harrison, D.J. 1992. United Kingdom offshore regional report: the geology of the southern North Sea. HMSO, London.

Carbajo A.E., Curto S.I., Schweigmann N.J., 2006, Spatial distribution pattern of oviposition in the mosquito Aedes aegypti in relation to urbanization in Buenos Aires: southern fringe bionomics of an introduced vector: Medical and Veterinary Entomology, v. 20, no. 2, p. 209-218.

Connor, D.W., Gilliland, P.M. Golding, N. Robinson, P., Todd, D., Verling, E. 2006. UKSeaMap: the mapping of seabed and water column features of UK Seas. Joint Nature Conservation Committee, Peterborough.

Cooper, R., Long, D., Doce, D., Green, S., Morando, A.. 2010. Creating and assessing a seabed sediment data layer for UKSeaMap 2010. British Geological Survey Commercial Report, CR/09/168. 
Costa, J.F., Zingano, A.C., Koppe, J.C. 2000. Simulation An Approach to Risk Analysis in Coal Mining. Exploration and Mining Geology 9, 43-49.

Cressie, N., Hawkins, D., 1980, Robust estimation of the variogram. Journal of the International Association of Mathematical Geology, v. 12, no. 2, p. 115-125.

Department for Environment, Food and Rural Affairs (U.K.) 2010. Charting Progress 2. The state of UK seas. Available at http://chartingprogress.defra.gov.uk/

Deutsch, C.V. \& Journel, A.G. 1992. GSLIB Geostatistical Software and User's Guide. Oxford University Press, New York.

Fannin, N.G.T. 1989. Offshore investigations 1966-87. British Geological Survey Technical Report WB/89/2.

Folk, R.L. 1954. The distinction between grain size and mineral composition in sedimentary rock nomenclature. J. Geol. 62, 344-359.

Gatliff, R.W., Richards, P.C., Smith, K., Graham, C.C., McCormac, M., Smith, N.J.P., Long, D., Cameron, T.D.J., Evans, D., Stevenson, A.G., Bulat, J.and Ritchie, J.D. 1994. United Kingdom offshore regional report: the geology of the central North Sea. HMSO, London.

Harris P., Baker, E. (in press). Why map Benthic Habitats? in (eds.) Harris, P. and Baker, E. Seafloor Geomorphology as Benthic Habitat; GEOHAB Atlas of seafloor geomorphic features and benthic habits, Elsevier. 3-22.

Howell, K.L. 2010. A benthic classification system to aid in the implementation of marine protected areas networks in the deep/high seas of the NE Atlantic. Biological Conservation $143,1041-1056$.

James, J.W.C., Pearce, B., Coggan, R.A., Arnott, S.H.L., Clark, R., Plim, J.F., Pinnion, J., Barrio Frójan, C., Gardiner, J.P., Morando, A., Baggaley, P.A., Scott, G., Bigourdan, N. 2010. The South Coast Regional Environmental Characterisation. British Geological Survey Open Report OR/09/51: 249.

Journel, A.G., Huijbregts, C.J., 1978, Mining Geostatistics. Academic Press, London. 
Lark, R.M. 2000. A comparison of some robust estimators of the variogram for use in soil survey. European Journal of Soil Science. 51, 137-157

Lark, R.M. 2003. Two robust estimators of the cross-variogram for multivariate geostatistical analysis of soil properties. European Journal of Soil Science, 54, 187-201.

Lark, R.M., Bishop, T.F.A. 2007. Cokriging particle size fractions of the soil. European Journal of Soil Science. 58, 763-774.

Lark, R.M., Papritz, A. 2003. Fitting a linear model of coregionalization for soil properties using simulated annealing. Geoderma, 115, 245-260.

Lark, R.M., Cullis, B.R., Welham, S.J. 2006. On spatial prediction of soil properties in the presence of a spatial trend:- the empirical best linear unbiased predictor (E-BLUP) with REML. European Journal of Soil Science. 57, 787-799.

Long, D., 2006. BGS detailed explanation of seabed sediment modified Folk classification. MESH (Mapping European Seabed Habitats) available at http://www.searchmesh.net/PDF/GMHM_Detailed_explanation_of_seabed_sediment_classification.pdf

Martín-Fernandéz, J.A., Thió-Henestrosa, S. 2006. Rounded zeros: some practical aspects for compositional data. In (Eds) Buccianti, A., Mateu-Figueras, G., PawlowskyGlahn, V. Compositional Data Analysis in the Geosciences: From Theory to Practice. Geological Society of London Special Publications 264, 191-201.

Ma, Y., Genton, M.G. 2001. Highly robust estimation of dispersion matrices. Journal of Multivariate Analysis, 78, 11-36.

Maravelias, C.D. Haralabous, J. 1995. Spatial distribution of herring in the Orkney/Shetland area (Northern North Sea): a geostatistical analysis. Netherlands Journal of Sea Research, 34, 319-329.

Matheron, G. 1963. Principles of geostatistics. Economic Geology 58, 1246-1266.

McBreen, F., Askew, N., Cameron, A., Connor, D., Ellwood, H., Carter, A. 2011. UK SeaMap 2010 Predictive mapping of seabed habitats in UK waters, JNCC Report 446. 
Payne, R.W. (ed) 2010. GenStat ${ }^{\circledR}$ Release 13 Reference Manual. Part 3 Procedure Library PL21. VSN International, Hemel Hempsted.

Pawlowsky-Glahn, V. \& Olea, R.A. 2004. Geostatistical Analysis of Compositional Data. Oxford University Press, New York.

Rawlins, B.G., Webster, R., Lister, T.R. 2003. The influence of parent material on topsoil geochemistry in eastern England. Earth Surface Processes and Landforms, 28, 13891409.

Sheather, S. J., Jones, M. C. 1991. A reliable data-based bandwidth selection method for kernel density estimation. Journal of the Royal Statistical Society series B, 53, $683-690$.

Webster, R. and Oliver, M.A. 2007. Geostatistics for Environmental Scientists, 2nd Edition. John Wiley \& Sons, Chichester.

Weltje, G.J., Robinson, S. 2012. Numerical methods for integrating particle-size frequency distributions. Computers and Geosciences, 44, 156-167.

Wentworth C.K. 1922. A scale of grade and class terms for clastic sediments. Journal of Geology 30, 377-392.

Zimmermann, B., Zehe, E., Hartmann, N.K., Elsenbeer, H., 2008. Analyzing spatial data: An assessment of assumptions, new methods, and uncertainty using soil hydraulic data. Water Resour. Res., 44: W10418 
Table 1. Summary statistics for data on the percentages of gravel, mud and sand, and on the ALR-transform of the gravel and sand contents.

\begin{tabular}{lrrrrr}
\hline & Gravel & Mud & Sand & ALR-gravel & ALR-mud \\
& & & & & \\
\hline & & & & & \\
Mean & 14.65 & 12.54 & 72.81 & -4.12 & -3.55 \\
Median & 1.49 & 2.14 & 85.90 & -3.96 & -3.56 \\
Standard Deviation & 24.08 & 23.41 & 29.52 & 3.54 & 2.94 \\
Skewness & 1.81 & 2.41 & -1.02 & 0.00 & 0.16 \\
Quartile 1 & 0.08 & 0.40 & 54.6 & -6.79 & -5.16 \\
Quartile 2 & 19.05 & 10.58 & 97.06 & -1.30 & -1.83 \\
& & & & & \\
\hline
\end{tabular}


Table 2. Results from cross-validation.

\begin{tabular}{lrrrr}
\hline Statistic & \multicolumn{2}{c}{$\begin{array}{c}\text { Standard estimator } \\
\text { ALR-gravel }\end{array}$} & $\begin{array}{r}\text { Robr-mud } \\
\text { ALR-gravel }\end{array}$ & ALR-mud \\
& & & & \\
\hline Mean error & -0.015 & -0.007 & -0.012 & -0.005 \\
Mean standardized & 0.895 & 0.859 & 2.024 & 2.171 \\
squared error & & & & \\
Median standardized & 0.225 & 0.172 & 0.475 & 0.427 \\
squared error & & & & \\
\hline
\end{tabular}




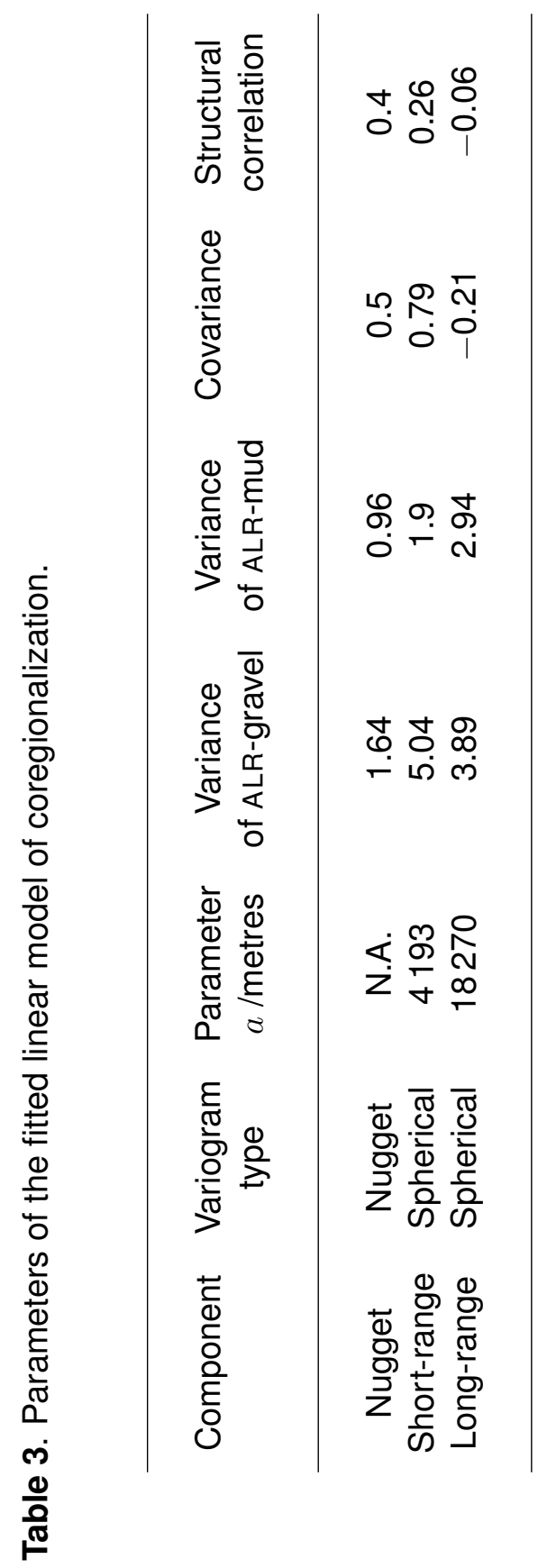


Table 4. Summary statistics on the probability of the class of maximum probability for 1500 randomly selected sites, divided into those where the observed class was the class of maximum probability and those where it was not.

\begin{tabular}{lcc}
\hline Statistic & $\begin{array}{c}\text { Sites where observed } \\
\text { class is not the } \\
\text { class of maximum } \\
\text { probability }\end{array}$ & $\begin{array}{c}\text { Sites where observed } \\
\text { class is the } \\
\text { class of maximum } \\
\text { probability }\end{array}$ \\
\hline Number of observations & 580 & \\
Mean* & 0.55 & 1420 \\
Median & 0.53 & 0.64 \\
Quartile 1 & 0.44 & 0.64 \\
Quartile 3 & 0.64 & 0.52 \\
Standard & 0.14 & 0.75 \\
deviation & & 0.14 \\
\end{tabular}




\section{Figure Captions}

1(a). Ternary diagram showing the four simplified sediment classes that are often used in habitat mapping (Long, 2006; Connor et al., 2006). (b) An alternative classification proposed by James et al. (2010).

2. Spatial distribution of sample sites where seabed sediment data are held by BGS.

3. Effect of the value of $\delta_{\mathrm{r}}$ for imputing non-zero values on (top) the coefficient of variation and (bottom) the correlation coefficient between ALR-transformed gravel and mud content.

4. Robust point estimates of the variograms and cross-variogram of ALR-transformed gravel and mud content (symbols) and the fitted functions (lines) constituting the Linear Model of Coregionalization (parameters in Table 2).

5. Kernel-estimated density function of the standardized kriging errors (heavy line) with a robustly-fitted normal probability density function (fine line) for (top) ALR-transformed gravel content and (bottom) ALR-transformed mud content.

6. Local conditional probability that the texture class is (a) Coarse (b) Mixed (c) Mud and Sandy Mud (d) Sand and Muddy Sand and (e) the local class of maximum probability along with a category where DigiBath indicates that the substrate is rock.

7. Probability of the class of maximum probability.

8. Estimated probability, from 2000 independent validation locations, that prediction sets of classes, defined increasingly broadly, include the observed class. Solid symbols: prediction sets are determined from the local probabilities computed from ALR-cokriging output. Open symbols: prediction sets are determined from the overall proportions of each class in the validation set.

9. For each class the proportion of observations belonging to that class in subsets of the 2000 validation sites defined by the 20th percentiles of the conditional probability of belonging to the class. The proportion is plotted against the mean probability in each subset. The straight line is the bisector: $x=y$. 
10. The class of maximum probability (a) and the probability of the class of maximum probability (b) according to the classification of James et al. (2000).

11. UK SeaMap seabed texture sediment map produced with the methodology described by Cooper et al. (2010) according to the classification of Long (1996) along with category 'rock and reef'. 
(a)

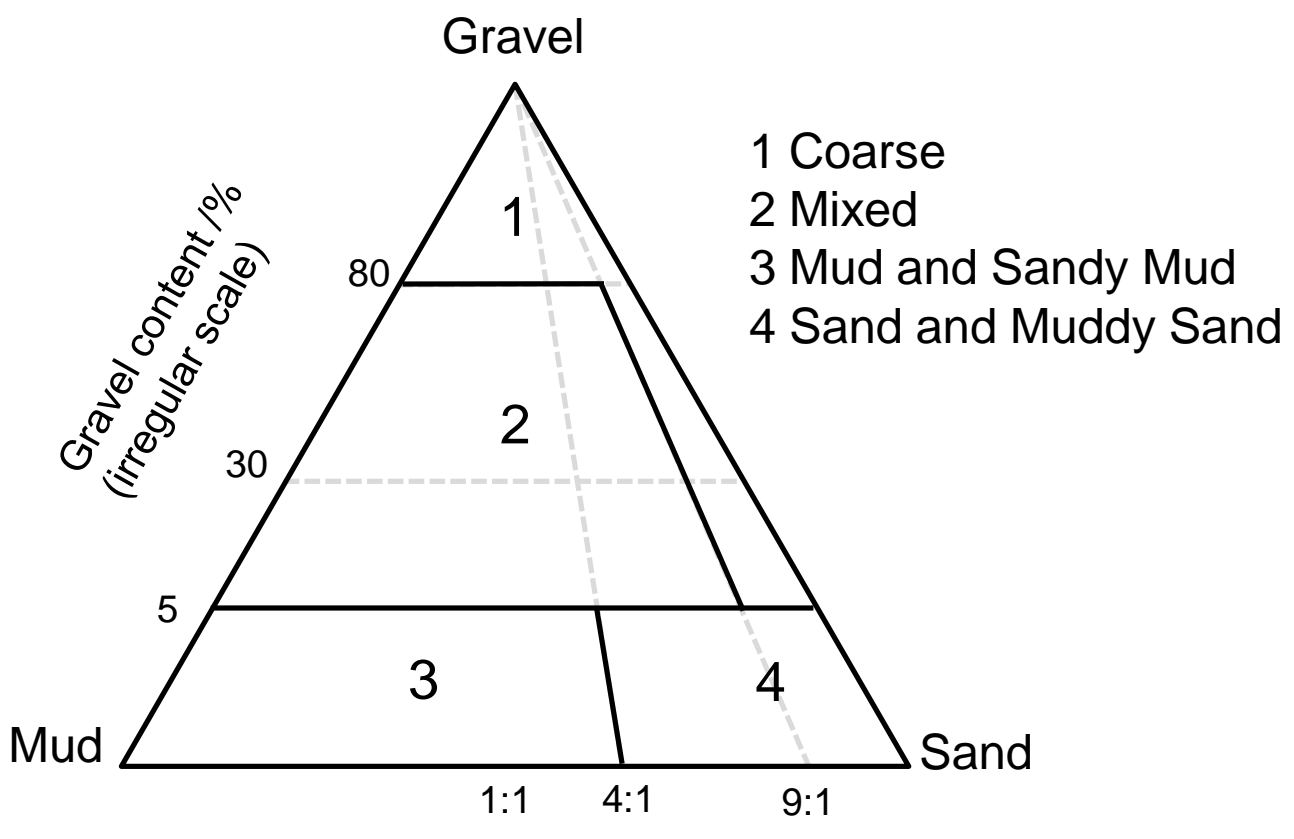

Sand: Mud ratio

(irregular scale)

(b)

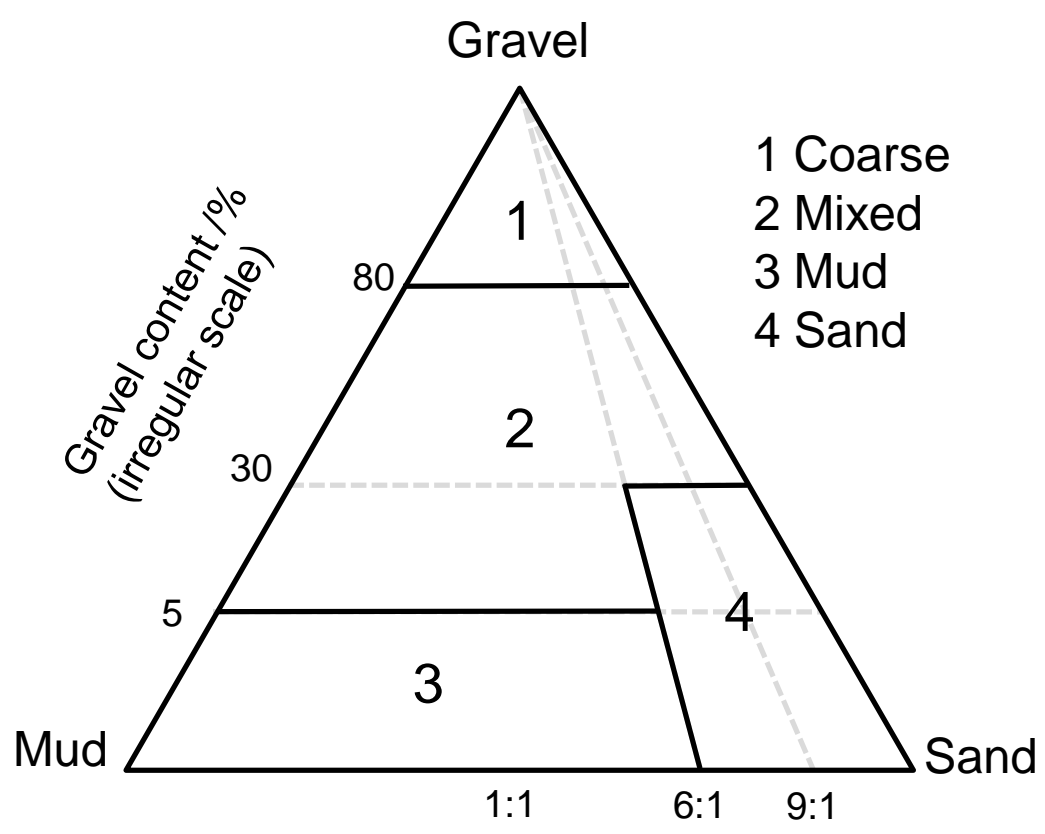

Sand: Mud ratio

(irregular scale) 


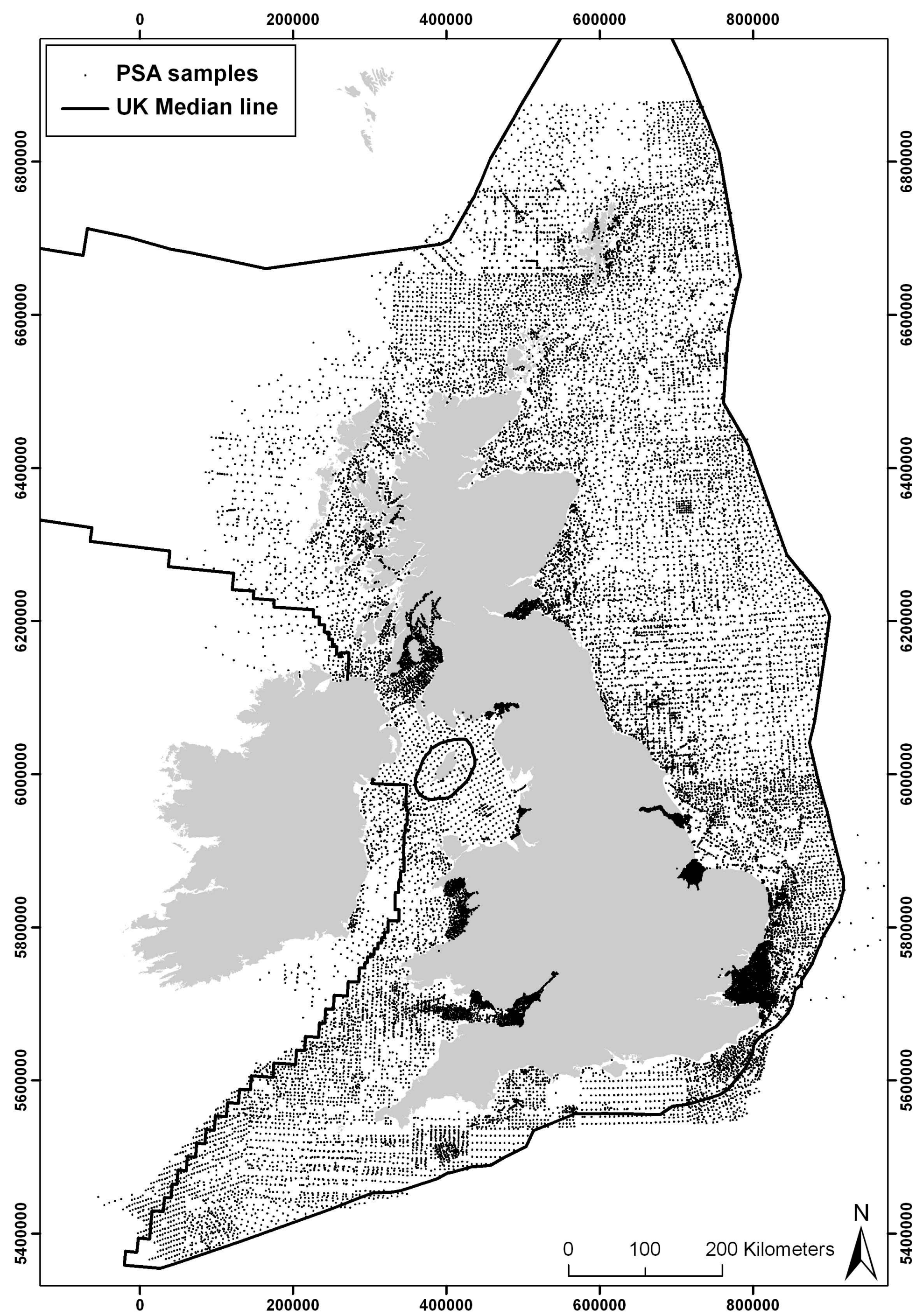




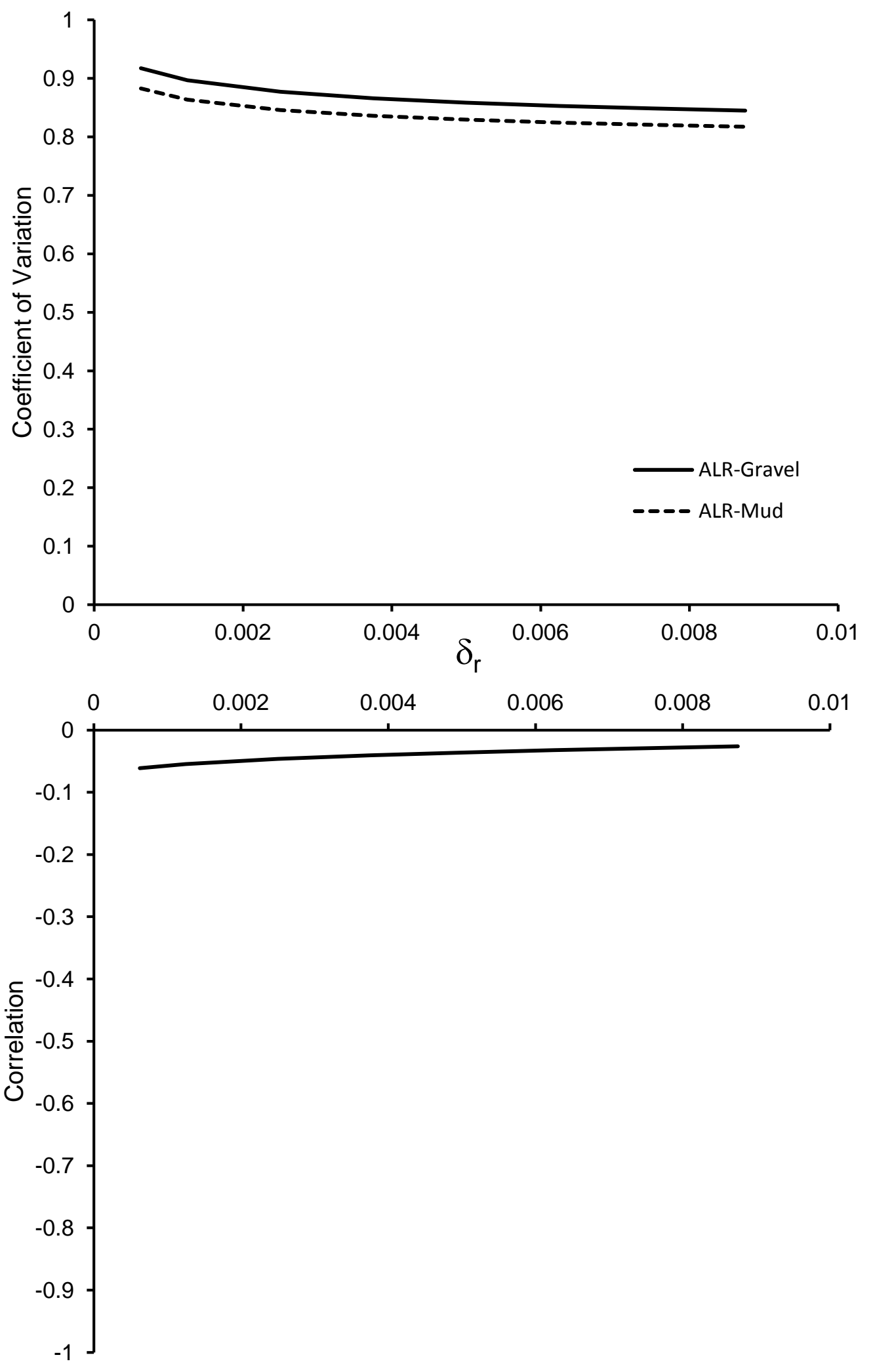




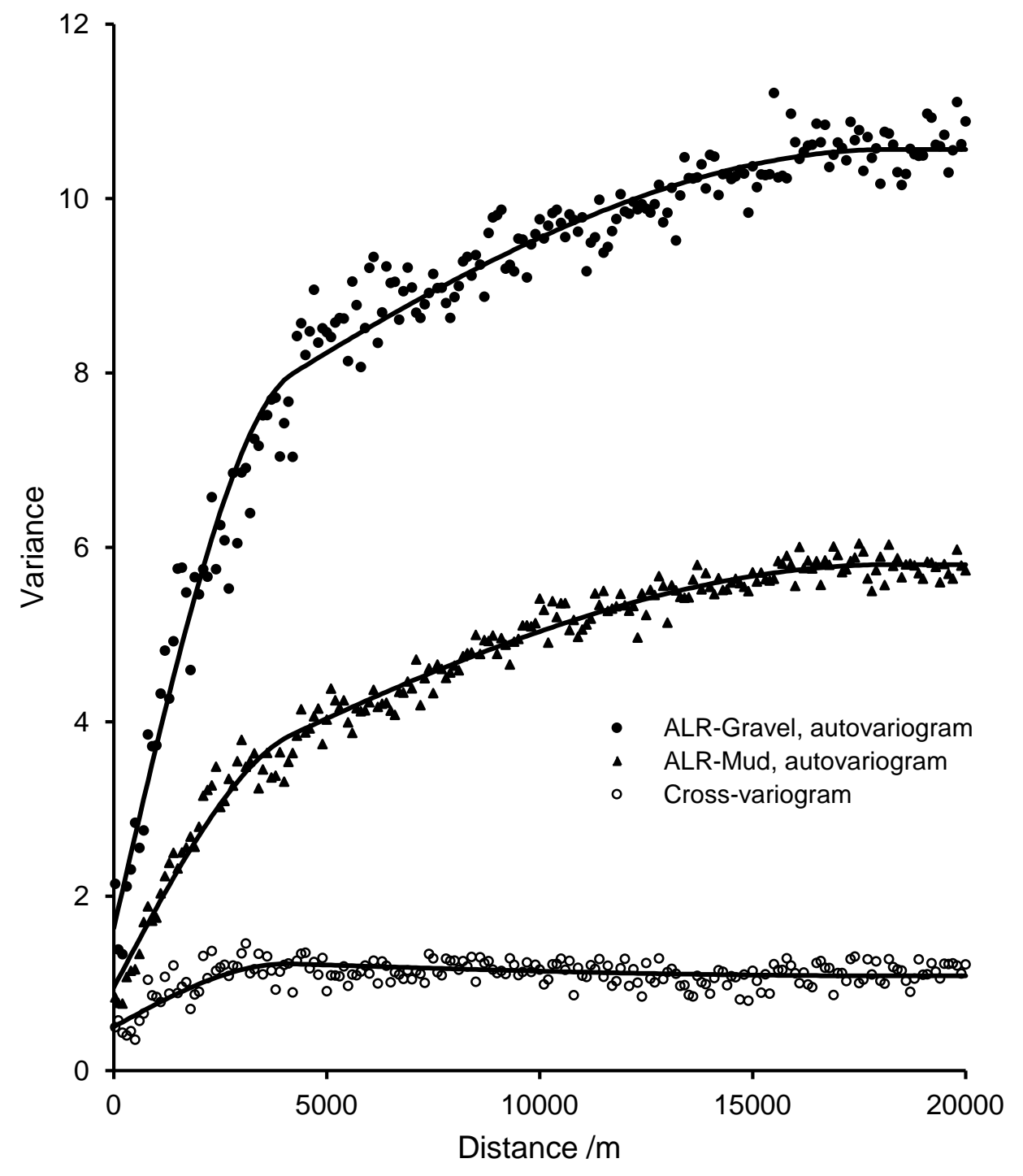



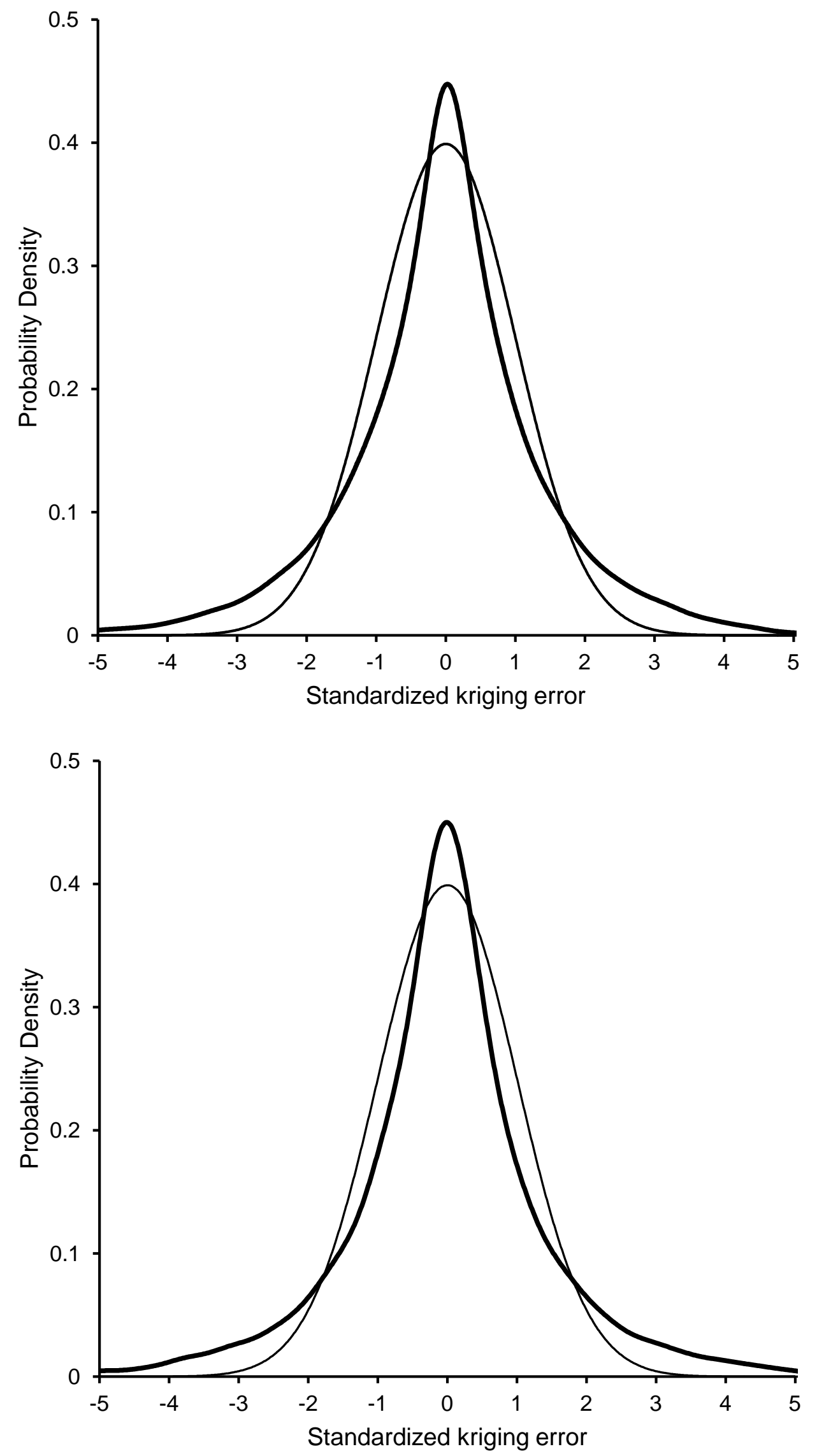
Probability of Class 1 (Coarse) 
Probability of Class 2 (Mixed) 
Probability of Class 3

6800000

6600000

6400000

6200000

6000000

5800000

5600000

5400000

(Mud and Sandy Mud)

0.00 to 0.16
0.16 to 0.33
0.33 to 0.49
0.49 to 0.65
0.65 to 0.82
0.82 to 0.98



+ Coarse

+ Mixed

+ Mud and sandy mud Sand and muddy sand + Rock (as in DIGIBATH) 



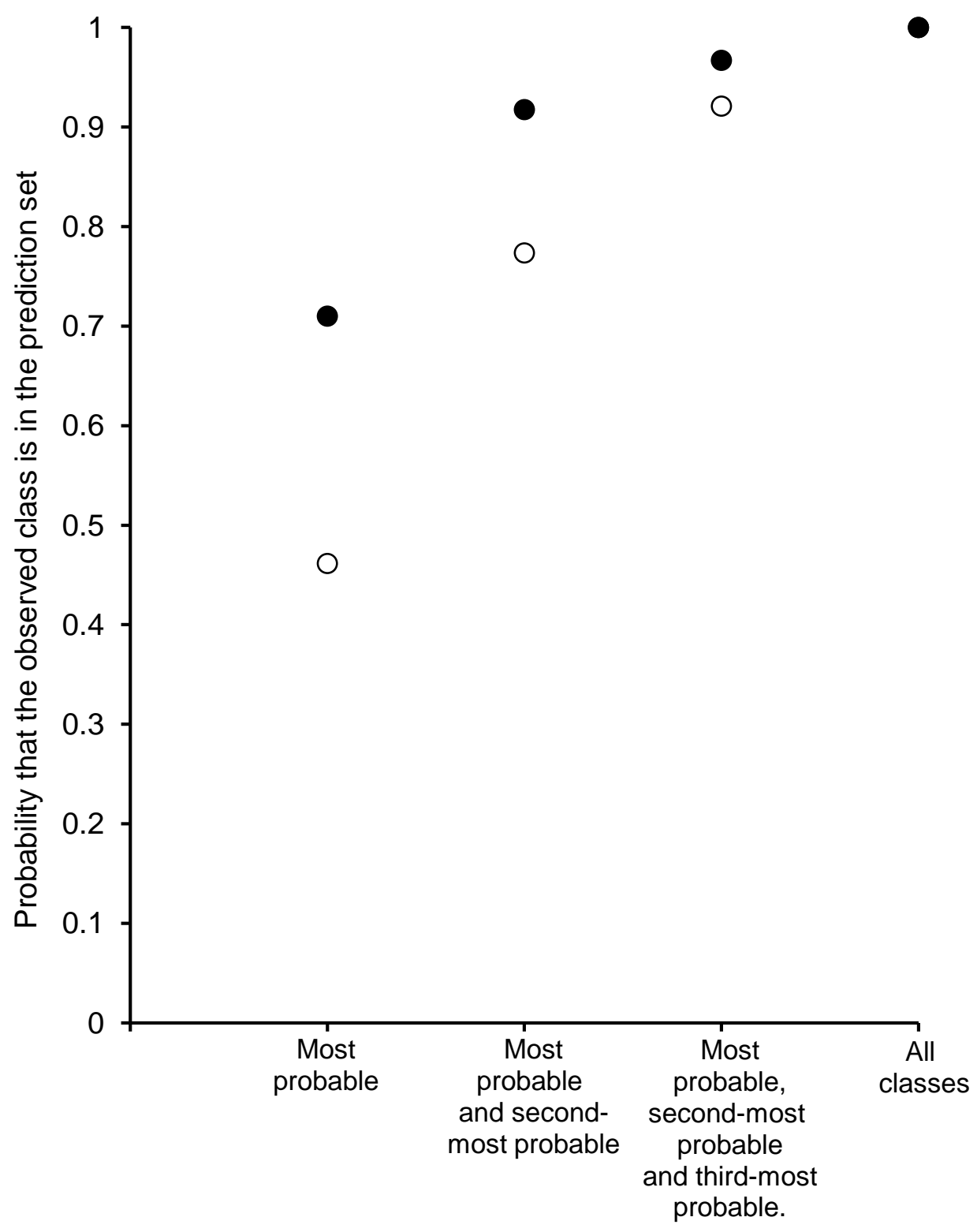

Prediction set 

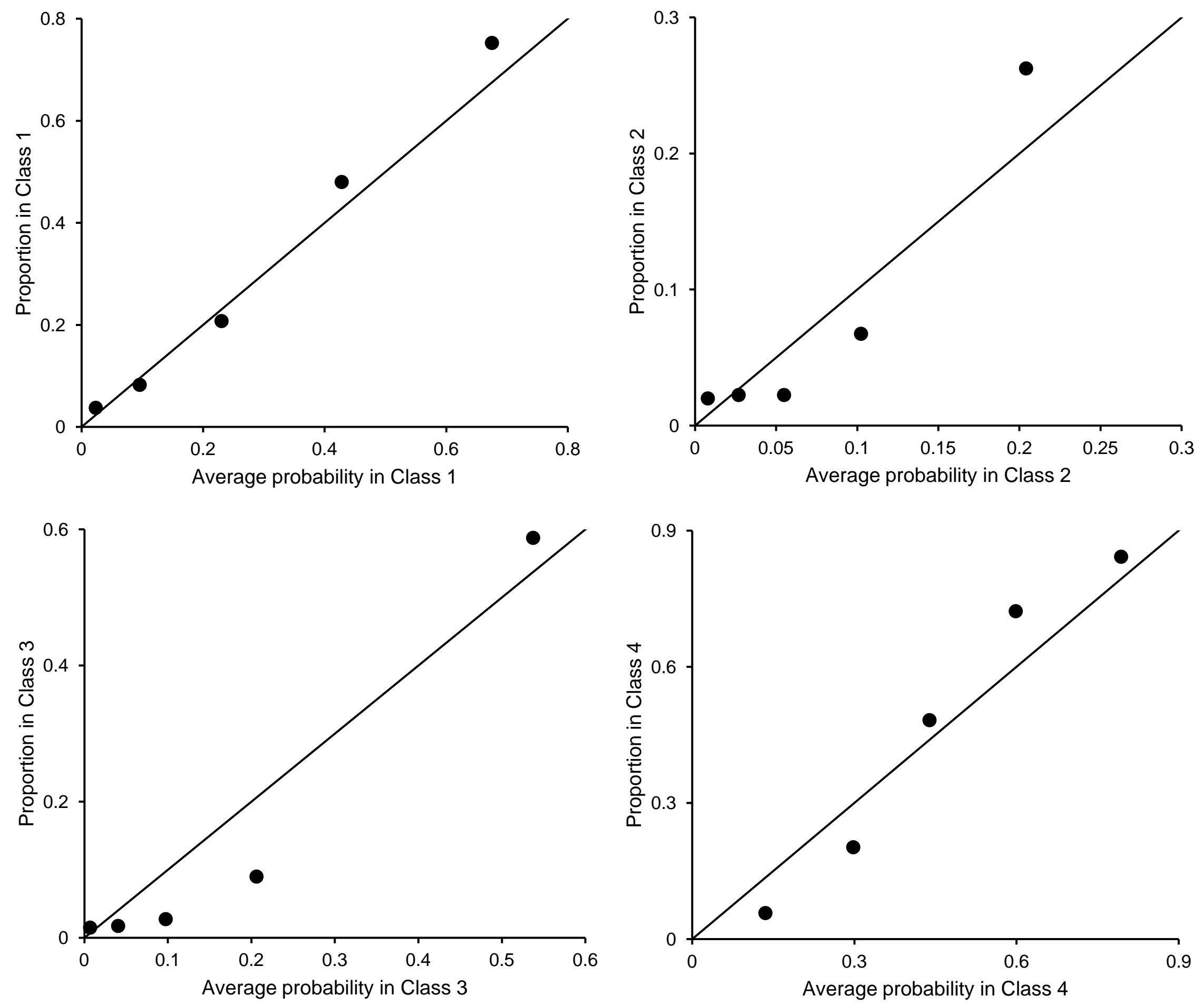
+ Coarse

+ Mixed

+ Mud Sand

+ Rock (as in DIGIBATH) 



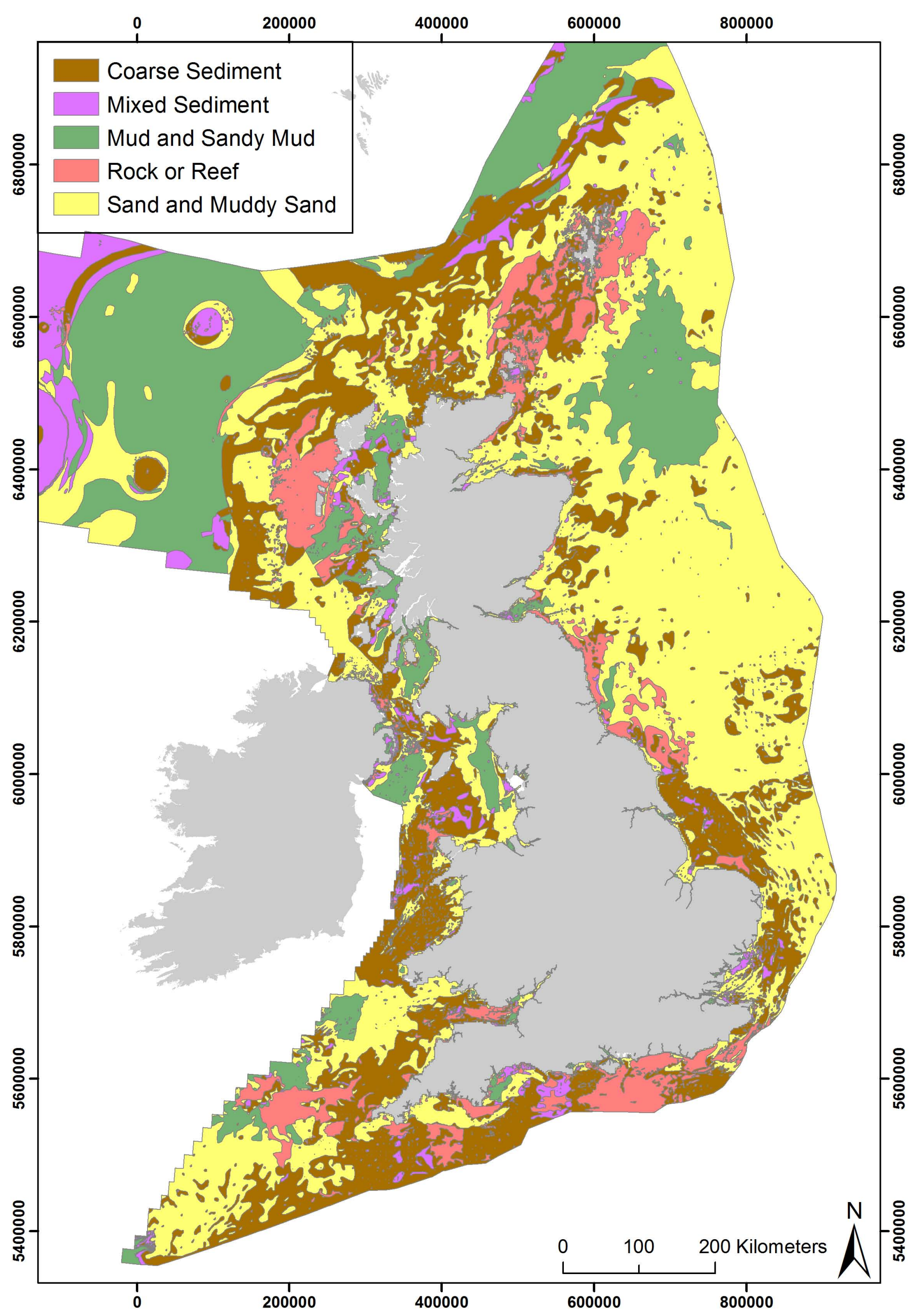

